\title{
How to Pick a Good Surgeon
}

\author{
Srinath Kamineni ${ }^{1 *}$, Eric Abbenhaus ${ }^{1}$, David Kirn ${ }^{2}$, Vikas Dhawan ${ }^{1}$ and Rick Papandrea ${ }^{3}$ \\ ${ }^{1}$ Department of Orthopaedic Surgery and Sports Medicine, University of Kentucky Elbow Shoulder Research Center, USA \\ ${ }^{2}$ Department of Plastic Surgery, University of Kentucky, USA \\ ${ }^{3}$ Department of Orthopaedic Associates of Wisconsin, Medical College of Wisconsin, USA
}

*Corresponding author: Srinath Kamineni, Department of Orthopaedic Surgery, Elbow Shoulder Research Centre, University of Kentucky, USA

\begin{abstract}
A shoulder surgeon has a shoulder injury and requests a specific shoulder surgeon colleague to operate. Instead, the patient is sent to an expert shoulder surgeon with an international reputation who delivers many lectures and writes numerous papers and book chapters. Surgery is performed but the shoulder gets infected and the overall result is unsatisfactory. After a legal challenge, the patient is allowed to go to the surgeon originally requested based on the patient's own insights into the profession. Revision surgery is performed and iatrogenic damage is discovered from the first surgery. However, the result of this revision surgery is good. This anecdotal case contains many aspects of healthcare decision making, control, and culpability which will be further elucidated here.
\end{abstract}

Keywords: Picking a Surgeon; Good Surgeon

\section{Introduction}

I am sure many of us have either heard or been asked the question, "How do you pick a good surgeon". Of all the academic questions we deal with on a daily basis as surgeons/physicians, this question perplexes us beyond our educational background due to the multitude of types of information needed to truthfully answer. Now, consider how hard this decision is to make for the lay person/patient who does not have the same insights into the inner workings of the profession. Before being able to pick a good surgeon, we need to define what makes a good surgeon. A surgeon is a medically trained doctor who has chosen to specialize in pathologies that can be treated with a surgical option but also has the knowledge and ethics to know when to treat non-surgically. However, most pathologies have a spectrum ranging from near normal to end-stage and management options that often depend on several factors: stage of the pathology, chronological versus physiological age, activity level, co-morbidities, availability of resources, etc. Therefore, anyone dealing with a specific pathology (e.g. osteoarthritis or shoulder instability) should not only understand the whole spectrum of the pathological process and its variants, but also be adept at managing these different stages with "stage specific solutions" instead of employing a "one operation fits all" philosophy. Picking a surgeon for oneself or one's family has several pathways, some controlled by the patient, and some by the agency responsible for the financial settlement.

There are no standard pathways for this choice, but when trying to understand what factors are of influence, we are able to identify many.

\section{Commonly Used Factors When A Patient Seeks A Surgeon}

a) A primary caretaker (family member/family doctor/ friend) recommends a surgeon due to personal experience or word of mouth recommendation [1,2]. While this sort of recommendation can be based on limited interactions with the surgeon, and his/ her outcomes, someone who has had a prolonged interaction would be able to gain a better insight into the outcomes from that surgeon. However, when limited interactions are the basis of a recommendation, this may prove to be detrimental to the patient since, for example, a surgeon who once performed a successful carpal tunnel surgery may not be the best option for performing a shoulder replacement.

b) An insurance company/workers compensation board will limit choice from surgeons within their approved network 
based upon whom they have an agreed terms and conditions contract [3]. This network is not based purely on quality of surgical skill, but is more based on a financial agreement with the insurer [3]. The financial entity does not have the responsibility of auditing the surgeon's outcomes, and hence can rarely make the choice easier for the patient except to limit the choice to a specific group of surgeons.

c) A surgeon with a higher rating on social media platforms is viewed as a better option than one with a lower rating [4]. Very few people understand how social media ratings come about. Individual ratings tend to be either strongly positive or strongly negative. Unhappy patients are more likely to complain, and thus there is a bias toward negative spontaneous social media ratings. Social media provides a platform from which the angry patient can voice their discontent. Happy patients are generally more interested in getting on with their lives following a successful surgery than devoting a lot of time to grandstanding on social media. Conversely, many well-run private practices are very efficient at collecting ratings from happy patients while avoiding collecting ratings from unhappy patients in their clinics. The latter practice may balance the equation by counteracting the negative bias or perhaps tilt responses to excessively positive. Social media ratings by patients are also more determined by their overall customer experience than their surgical outcome. Factors such as wait time, parking, office atmosphere, and interpersonal interactions with the surgeon and staff have been shown to have a stronger impact on social media ratings than quality of the outcome. This is logical since humans have daily interpersonal interactions which provide a standard for comparison, unlike a surgical procedure which is generally limited to a small number of times in an individual's life. Hence, social media may focus on extraneous factors and can be manipulated. Thus, online patient ratings are not the best indicator of a good surgeon. If every patient had to input a rating, usefulness might be improved and this may represent a direction for the future.

d) Speed of Surgeon [5]. Is a slow surgeon better than fast surgeon, or vice versa? Some surgeons have a reputation for being very quick while others have a reputation for being very slow, or sometimes described as meticulous. Inorder to fully appreciate this issue, one needs to understand what a particular pathology requires in order to resolve the symptoms. Taking a very simple example, carpal tunnel release, some surgeons take 4 minutes to complete this through a $1 \mathrm{~cm}$ incision, versus others take 90 minutes or more with a $5 \mathrm{~cm}$ incision or an endoscopic technique. While a fast surgery may miss some steps, although all being achievable for a carpal tunnel surgery within the 4 minutes, the same surgery with a larger incision and a long operative time may have a greater risk of infection and scarring, due to many more surgically performed strokes of the knife. The latter refers to the surgeon performing more surgery than is necessary to achieve the result, under the label of being "meticulous" and "precise". Hence, performing more surgery than is necessary to treat the pathology is detrimental to the outcome. Therefore the speed of the surgeon is not always easy to understand without the context of the surgical results achieved by that surgeon.

e) Communication skills [6].A surgeon who can communicate well is better than a surgeon who is not a good communicator [6]. Communication is a central and under-valued aspect of any surgeon's skill set. The most important factor in healthcare is the needs of the patient. Hence, it is the patient who needs to understand the problem, and how to fix it, to a level compatible with his/her educational background. Communication between the surgeon and the patient allows the necessary information to be patient-centric, in order that confidence is built within that relationship [6]. A surgeon who is able to communicate well with all the patient demographics and educational levels in his/her practice should be viewed in a positive light, while the abrupt, minimal communicator cannot justifiably impart the necessary information. If a patient is not made to understand the whole process, he/she cannot be an effective part of the treating team, and patients should be considered as part of the team.

f) Academic impact [7]. "A surgeon who writes a lot of papers is better than one who doesn't write papers". Surgery, like any athletic pursuit or manual trade (carpenter, motor mechanic, etc.) is a dexterous skill, combined with knowledge and the translation of that knowledge to the physical activity. As any athlete knows, it takes a very long time to train one's body to perform a physical task at a certain level, more than $90 \%$ of their time. So if an athlete spends a significant amount of time reading about a skill, and less time translating the skill into his/her own physical performance, the results are failure in competition. Surgeons are no different. Those who spend significant amounts of their time in research, giving talks, writing papers, advocacy and committee work, etc. have less time to see and diagnose patients, operate and treat patients, follow up and assess patients' outcomes etc. Although there is not a welldefined parameter to judge the optimal times for cerebral versus dexterous activities, the principle of the athlete is relevant to the surgeon.

g) Education at high profile institutions [7]. Those with educational backgrounds in high profile institutions are considered with greater regard than those with lesser academic profile institutions [7]. It should be constantly remembered that surgery is an intricately intermingled cerebral and dexterous skill. Being overweight in either may not compensate for achieving the best results. High profile institutions achieve their profile by many different means, but none achieve it by the quality of the training they provide to the next generation of surgeons. The institutions select their surgeons based on qualities important to the mission of the institution, and it can be assumed the trainees who are trained there will both actively and passively achieve some of those qualities. However, great institutions can produce poor quality surgeons and lesser institutions can produce outstanding surgeons so it is not a guaranteed assurance to rely on the surgeon's educational institution's reputation. 
h) Specialty Board Certification [8]. The American Board of Medical Specialties (ABMS) and its 24 member boards are organizations established to ensure consistency of training and practice within established guidelines for surgical specialists. These organizations seek to ensure that surgeons have a specific knowledge base and have completed requisite numbers of surgical procedures during their training. In certain competitive areas such as cosmetic surgery, physicians lacking ABMS certification seek to deceive prospective patients by creation of non-ABMS boards which do not require any specific training and allow surgeons to join simply by paying a fee. In orthopaedic surgery the ABOS (American Board of Orthopaedic Surgery) seeks to ensure this quality with didactic exams and a review of cases performed by the surgeon. While these boards try to ensure quality, they do not have the capacity to be stringent enough and would require considerable investment to improve quality of candidates. So a "Board Certified" surgeon may not always be a better choice than a non-board certified surgeon, but the certification ensures a minimum quality.

i) Availability of the surgeon [9]. Some consider a surgeon who is easily available for new appointments to be better than those who are really difficult, or have a very long waiting list. Similar to a restaurant, would you prefer to eat at an empty restaurant or one where it is always busy? The three 'A's of medical practice priority used to be (and should be) "Ability, Affability, Availability". Unfortunately, with the societal shift in attitudes the order has reprioritized to "Availability, Affability, Ability" [9].

j) Intellectual honesty. One of the most important qualities for a medical professional is the ability to honestly assess one's own level of knowledge and skill. Not all surgeons are equal, just like not all tennis players or restaurants are equal. Hence, when a surgeon is unable to perform a certain operation that may be the optimal treatment for a particular patient, it takes intellectual honesty for that surgeon to communicate this discrepancy and refer the patient to another surgeon who is able to perform that optimal procedure. It is dishonest to perform a procedure that is not in the best interest of the patient just because it is a procedure that the surgeon is able to perform, regardless of the suitability of the performed procedure to the presenting pathology. An example of this is when surgeons perform reverse shoulder replacements instead of anatomical total shoulder replacements despite the presence of all the factors that can make an anatomical replacement successful. Although a reverse replacement is an easier procedure to perform, it sacrifices an intact rotator cuff. The price of an inappropriate operation is paid at a later date by the patient, with very fewer good options if the reverse shoulder replacement fails.

k) Volume and institutional protocols [10]. Frequent performance of a procedure by a surgeon naturally leads to technique refinements if the surgeon is thoughtful about the process. Likewise, the operating room staff and other associated individuals such as anesthesiologists become familiar with the surgeon's routine and optimize their functions accordingly. Further, some surgical procedures benefit from ancillary services such as specialized nursing care, physical therapy or occupational therapy working in a coordinated manner immediately following the procedure. In these instances, the good surgeon's skills are only one component of a successful surgical outcome, albeit an important one. Surgeons who perform low volumes of surgery are likely to be less dexterous than those who perform high volumes of surgery, with a required debate regarding the definition of high, low, and adequate volumes to maintain dexterous skills.

l) Word of mouth reputation [1,2]. The natural course of surgical practice is that the surgeon reaps what he sows. That is, if a surgeon performs an operation successfully on one patient, that patient is more likely to tell their friends and their referring physician about their experience [1,2]. Those patients and providers are more likely to send other patients, and thus forms the basis of "word of mouth" referenced in the first paragraph. This leads to a snowball effect on the surgeon's practice which grows over the course of a career. Name recognition for surgeon grows over time and hence the effect of word of mouth becomes more powerful for them individually. Name recognition would seem to imply a larger pool of satisfied patients, but this is not always true as other factors can lead to name recognition such as advertising. Further, it is likely this effect is more powerful in smaller communities with fewer providers compared to larger communities.

m) Access to specialized equipment [10]. Specialized equipment may allow an appropriately-skilled surgeon the ability to perform a procedure in a less invasive, safer, or more efficient manner. The recent innovations in robotic surgery provide an example for certain procedures. However, it is important to note that just because a technology is new or "hi-tech", it is not necessarily better or safer than established methods. For instance, endoscopic carpal tunnel release has no superior data compared to minimal incision release. Furthermore, technology presents a constantly changing environment and yet it can take years for a particular innovation to mature to its fullest potential or fade out.

n) Advertising [11]. For elective surgical procedures, intense marketing efforts are exerted by some surgeons. These may take the obvious forms of billboards or newspaper advertisements, but may also be more subtle such as marketing on social media platforms. One such example in the plastic surgery world is RealSelf. Here, surgeons subscribe to the platform and participate in interactions with users of the site. Although specialty societies and medical

ethics in general prevent surgeons from claiming they are "the best" at a particular procedure, many advertising modalities purport those messages in a thinly veiled manner. Advertising is rarely based on any quantifiable measures and its reach is only limited by the amount of money the surgeon wishes to spend to promote themselves. In general surgery, orthopaedic

surgery, etc., "Best Doctor" lists are more an exercise in networking than the ability of the doctor or his/her outcomes. For this reason, these lists should not be blindly accepted as a quality control. 
o) Complications and quality metrics [12]. Government and institutions have an interest in measuring surgeon quality but they too have found this difficult [12]. Comparing providers by their mortality rate or rate of specific complications are frequently used metrics [12]. However, these may not accurately portray a surgeon's quality or skill [12]. For example, the heart surgeon who takes on the most difficult cases will naturally have a higher complication rate due to the systemic disease in those sicker patient populations; a shoulder surgeon who takes on the multiple-operation revision case may have lesser outcomes than those who cherry-pick the simplest cases and avoid the difficult ones; the general surgeon who accepts the unhealthy "botched" hernia repair patient may have longer hospital stay data than those operating on the primary hernia repair in a healthy patient, etc.

\section{Objective Parameters that are Difficult to Quantify}

a. Doctor's Knowledge of the pathologies in his/her scope of practice

b. Ability in the skills that make up surgery (dextrous and cognitive)

\section{c. Complications in easy and difficult cases}

d. Ability to treat unexpected and complication outcomes

In the ideal world we should be able to advise patients according to a surgeon's objective outcomes (listed above) as well as patient's subjective scoring. If this data is available for all surgeons and all patients treated, a much more accurate system will exist. Sadly very few registers exist, although joint registries are becoming more widespread. However, there are so many more operations than just joint replacements and registries are only as good as the inputted data as they rely on how honestly and extensively the data is entered and interpreted. At present, a patient being referred to a surgeon needs to be aware of all the factors discussed above, and ask direct questions of the surgeon:

\section{a) What is my problem}

b) What are the treatment options, and why do you recommend what you are recommending?

c) How many such operations have you done?

d) How many complications have you had?

e) How did you treat the complications, adverse events, etc. These questions should allow a patient to better understand the surgeon and surgeon's recommendation.

After patients understand their problem, they can begin to understand the different options of fixing the problem. The different treatment options, as well as the expected outcomes and potential issues, should be thoroughly explained to the patient. This allows for a dialogue between patient and surgeon where the patient's expectations can be communicated and incorporated into the treatment plan. By asking the surgeon to provide a reason for the recommended treatment, the patient can ensure that the surgeon is taking into account personal factors including the patient's medical conditions, financial situation, or desires. Once the patient is satisfied that the surgeon has recommended a good treatment plan for them, they should ask about the surgeon's experience with this treatment. The number of procedures performed, as well as the number and severity of complications, should be asked by the patient. This serves to further provide improved mutual understanding between the patient and surgeon about expected outcomes. A surgeon has the responsibility to accurately and honestly answer such questions, since it remains an honor for the surgeon that the patient has chosen to entrust him/her with their health. Each of these questions is important as, for example, surgeons who only rarely perform a procedure may have never experienced a complication whereas a very experienced surgeon, who will often deal with a more complex spectrum of pathology and possibly revision cases of failed surgeries, may have had more complications. Furthermore, the severity of the complications is of utmost importance as these may have very different clinical implications.

By taking into account the options for treating the problem, as well as the surgeon's ability and past experiences with the operation(s), patients will better understand their expected outcome. However, a patient asking these questions also can help the surgeon better understand the patient and their goals of treatment. As a result, this dialogue generates an improved mutual understanding between the patient and surgeon, potentially leading to better outcomes. After a patient has received truthful and satisfactory answers to these questions, they can be assured that they have picked a good surgeon. Until there is a time where a publicly accessible registry of all operations and treatments carried out, along with outcomes and complications, is available, the open and honest communication between a patient and surgeon remains of utmost importance.

\section{References}

1. $\mathrm{Lu} \mathrm{N}, \mathrm{Wu} \mathrm{H}$ (2016) Exploring the impact of word-of-mouth about Physicians' service quality on patient choice based on online health communities. BMC Med Inform Decis Mak 16(1): 151.

2. Barnett ML, Keating NL, Christakis NA, OMalley AJ, Landon BE (2012) Reasons for choice of referral physician among primary care and specialist physicians. J Gen Intern Med 27(5): 506-512.

3. Kyanko KA, Curry LA, Busch SH (2013) Out-of-network physicians: how prevalent are involuntary use and cost transparency? Health Serv Res 2013;48(3): 1154-1172.

4. Ventola CL (2014) Social media and health care professionals: benefits, risks, and best practices. P T 39(7): 491-520.

5. Reames BN, Bacal D, Krell RW, Birkmeyer JD, Birkmeyer NJ, et al. (2015) Influence of median surgeon operative duration on adverse outcomes in bariatric surgery. Surg Obes Relat Dis 11(1): 207-213.

6. Ha JF, Longnecker N (2010) Doctor-patient communication: a review. Ochsner J 10(1):38-43.

7. Abghari MS, Takemoto R, Sadiq A, Karia R, Phillips D, et al. (2014) Patient perceptions and preferences when choosing an orthopaedic surgeon. Iowa Orthop J 34: 204-208. 
8. Marsa Linda (2017) How to Pick the Right Surgeon. AARP, AARP.

9. Marshall DA, Deal K, Conner Spady B, Bohm E, Hawker G, (2018) How do patients trade-off surgeon choice and waiting times for total joint replacement: a discrete choice experiment. Osteoarthritis and Cartilage 26(4): 522-530.

10. Smith H, Currie C, Chaiwuttisak P (2018) Patient choice modelling: how do patients choose their hospitals? Health Care Manag Sci 21: 259.
11. Mohney S, Lee DJ, Elfar JC (2016) The Effect of Orthopedic Advertising and Self-Promotion on a Naïve Population. Am J Orthop (Belle Mead NJ) 45(4): 227-232.

12. Chou E, Abboudi H, Shamim Khan M, Dasgupta P, Ahmed K. (2015) Should surgical outcomes be published? J R Soc Med 108(4): 127-135.
This work is licensed under Creative

Commons Attribution 4.0 License

To Submit Your Article Click Here:

Submit Article

DOI: $10.32474 /$ OSMOAJ.2019.03.000153

$\begin{gathered}\text { Orthopedics and Sports Medicine } \\ \text { Open Access Journal }\end{gathered}$
Assets of Publishing with us
- Global archiving of articles
- Immediate, unrestricted online access
- Rigorous Peer Review Process
- Authors Retain Copyrights
- Unique DOI for all articles

